
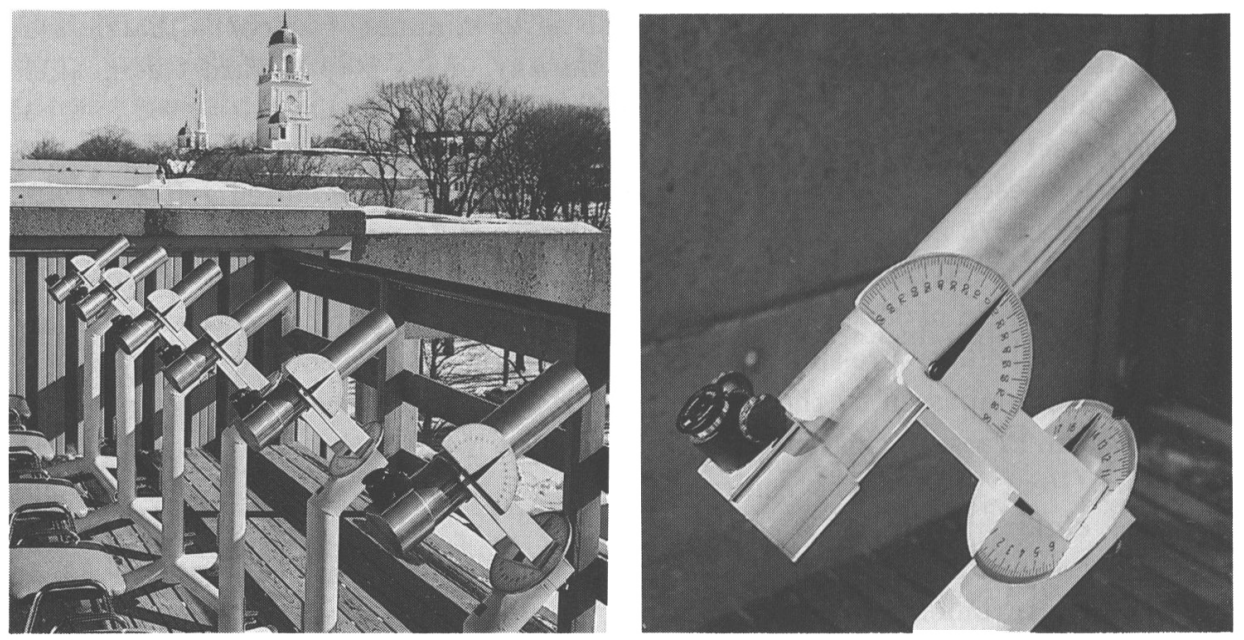

Fig. 1. Telescopes for students. Left: six telescopes mounted on the observing deck. Right: close-up view of one telescope.

\title{
References
}

Bishop, R.L. 1986, J. Roy. Astron. Soc. Can., 80, No. 4, 211-215.

\section{USING CCD'S IN INTRODUCTORY-LEVEL COLLEGE ASTRONOMY LABORATORIES}

Thomas J. Balonek

Colgate University, Dept. of Physics and Astronomy, Hamilton, New York 13346, U.S.A.

\section{The CCD and Interactive Image Display Systems}

Our university recently purchased a liquid-nitrogen-cooled CCD camera system (from Photometrics Ltd., Tucson, Arizona) which has been installed on our campus' 40-cm multiple access (Cassegrain/Newtonian) telescope. Images are reduced online at the observatory using Photometrics' microcomputer-based analysis software package, which includes operations for standard data acquisition and initial stages of data reduction - including corrections for bias, dark current, and flat fielding. Images are displayed on a 256-level-gray-scale black and white monitor. Additional post-processing can be done either on the CCD system's computer at the observatory 
or on IBM-AT/PC's located both at the observatory and in the laboratory.

Because of the ease of use of the PC-based program and the ready availability of PC's, we are using this system to develop laboratory exercises to be used in introductory astronomy courses. The eight-color image-display program, developed by Michael Newberry and Stephen Gregory (Institute for Astrophysics, University of New Mexico), has many features similar to other well known astronomical CCD image-analysis packages. For our research work, analysis is done on an IBM-AT clone, while IBM PC's and PC clones (equipped with a mouse and an EGA color graphics card with $250 \mathrm{k}$ RAM) will be used for introductory laboratory exercises. Images can be stored either on a hard disk or on a floppy (some images need to be "trimmed" in order to fit on a $360 \mathrm{k}$ floppy).

\section{Laboratory Exercises Under Development}

Laboratory exercises for use in our introductory astronomy courses are being developed by Colgate students (astronomy/physics majors), who are responsible for defining the scientific and educational goals of the project, obtaining the CCD images with our system, and writing the laboratory exercises.

Operation of the CCD system is demonstrated as part of the laboratory exercises, so that students experience the many steps involved in making accurate astronomical measurements. In addition to analyzing prints of several images, students use the microcomputer-based image-display program to deduce properties of the objects being studied.

Exercises under development include:

Enhanced images of galaxies in the Virgo Cluster. This exercise will contain images of several galaxies that are studied in the University of Maryland's laboratory exercise on galaxies ${ }^{1}$. We plan to have images of elliptical, spiral, and interacting galaxies that the students can study with the PC image display system. This exercise will require the student to learn techniques used in extracting quantitative results from high-dynamic-range images. Using these enlarged images (compared to the scale of the Palomar Sky Survey print images used in the Maryland exercise), students will be able to study the structure, brightness distribution, and density profile in different galaxy types, and to test conclusions that they had made in the earlier lab exercise.

Colors of stars and the H-R Diagram. Students will perform photometric reduction of an open cluster, determining the magnitude and color of stars. These results will be used in constructing a cluster $\mathrm{H}-\mathrm{R}$ diagram. This exercise will demonstrate the procedures involved in accurate calibration of images.

Planetary-satellite orbits. Students will investigate Kepler's laws of motion by measuring satellite movements. Images will be obtained of the Jupiter or Saturn systems during several satellite revolutions. By accurately measuring the positions

\footnotetext{
${ }^{1}$ Reprinted in Teacher's Guide for Contemporary Astronomy, 4th ed, by Jay M. Pasachoff (Saunders College Publishing, Curtis Center, Independence Square West, Philadelphia, PA 19106, U.S.A.; 1989).
} 
of the moons relative to the planet, students will be able to use real data to deduce Kepler's laws.

These exercises are being developed by our astronomy students under the direction of faculty. Thus, not only will introductory students use observations obtained with our CCD system, but also the astronomy majors will learn how to obtain accurate CCD images and how to develop useful laboratory exercises. Motivated students will be encouraged to undertake an observing project of their selection in which they participate in all the steps involved in selecting a suitable object to observe, followed by obtaining, calibrating, and analyzing the CCD image. It is our hope that students will be excited by using results obtained from a research-quality instrument that is located on our campus.

\title{
3. Acknowledgment
}

Funding for the purchase of the CCD system was provided by a National Science Foundation-College Science Instrumentation Program grant and by Colgate University. Support for student participation in developing laboratory exercises has been provided by a Sloan Foundation grant to Colgate University. Once development of these projects is completed, we expect to make our images and exercises available to interested educators. We welcome any comments or inquiries about our CCD system or laboratory exercises.

\section{MODERN PHOTOMETRY LAB EXERCISES FOR STUDENTS IN INTRODUCTORY ASTRONOMY}

\author{
Daniel B. Caton \\ Department of Physics and Astronomy, Appalachian State University, \\ Boone, North Carolina 28608 U.S.A.
}

Introductory astronomy lab exercises have traditionally been rather simple in nature - observations are often limited to visual observing, sketching, and perhaps photography. These types of labs are more like 19th-century astronomy than the techniques used by modern astronomers. Students should also be introduced to quantitative work in astronomy, in the same way that they are in equivalent introductory physics and chemistry labs. A typical solution to some of these problems has been to use published data (for example, the $S k y \mathscr{E}$ Telescope lab exercise series) to provide numerical work. This is less satisfactory for the student than obtaining his or her own quantitative data to reduce and analyze.

At Appalachian State University we have been working on the development of a set of modern lab exercises that address the needs discussed above. The first of 